\title{
Incidence comparison of adverse events in patients with inflammatory bowel disease receiving different biologic agents: retrospective long-term evaluation
}

\author{
Brigida Barberio ${ }^{1}$, Edoardo Vincenzo Savarino ${ }^{1}$, Timothy Card $^{2,3,4}$, Cristina Canova ${ }^{5}$, Francesco Baldisser ${ }^{1}$, \\ Alessandro Gubbiotti ${ }^{1}$, Davide Massimi ${ }^{1}$, Matteo Ghisa ${ }^{1}$, Fabiana Zingone ${ }^{1}$ \\ ${ }^{I}$ Division of Gastroenterology, Department of Surgery, Oncology and Gastroenterology (DISCOG), University of Padua, Padua, Italy; \\ ${ }^{2}$ NIHR Nottingham Biomedical Research Centre, Nottingham University Hospitals NHS Trust and University of Nottingham, Nottingham; \\ ${ }^{3}$ Nottingham Digestive Diseases Centre, University of Nottingham, Nottingham; ${ }^{4}$ Division of Epidemiology and Public Health, School of \\ Medicine, University of Nottingham, Nottingham, UK; ${ }^{5}$ Department of Cardio-Thoraco-Vascular Sciences and Public Health, University of \\ Padua, Padua, Italy
}

Background/Aims: Current literature is lacking in studies comparing the incidence of adverse events (AEs) in patients with inflammatory bowel diseases (IBD) treated with adalimumab (ADA) or vedolizumab (VDZ) in a real-life scenario. Therefore, our primary aim was to compare the AEs occurring in patients taking ADA to those of patients taking VDZ. Methods: In this single center study, data on AEs from IBD patients who underwent treatment with ADA and VDZ were retrospectively collected. AE rates per 100 person-years were calculated. A Cox regression model was used to estimate the hazard ratios of the AEs between the 2 drugs. Results: A total of 16 ADA patients (17.2\%) and 11 VDZ patients (7.6\%) had AEs causing drug interruption during the study period $(P=0.02)$. Most of the AEs were noninfectious extraintestinal events (50\% in ADA and $54.5 \%$ in VDZ) while infections accounted for $31.2 \%$ of the AEs in patients treated with ADA and $27.3 \%$ in those treated with VDZ. The incidence rate of AEs causing withdrawal of therapy was 13.2 per 100 person-years for ADA and 5.3 per 100 person-years for VDZ, corresponding to a $76 \%$ lower risk in patients in VDZ. Considering the first year of treatment, we observed 34 subjects treated with ADA (36.5\%) having at least 1 AEs and $57(39.3 \%)$ among those taking VDZ $(P=0.67)$. Conclusions: VDZ has a lower incidence rate of AEs causing withdrawal of treatment compared to ADA but a similar risk of AEs not causing drug interruption. Real-life head-to-head studies are still necessary to further explore the safety profile of these drugs. (Intest Res 2022;20:1 14-123)

Key Words: Inflammatory bowel disease; Biological therapy; Adalimumab; Vedolizumab; Adverse events

\section{INTRODUCTION}

Inflammatory bowel diseases (IBDs), including ulcerative colitis (UC) and Crohn's disease (CD), are chronic disorders of the intestinal tract characterized by relapsing and remitting intestinal inflammation and associated with a reduced quality of life. ${ }^{1}$

Received February 27, 2021. Revised April 29, 2021. Accepted June 16, 2021 Correspondence to Edoardo Vincenzo Savarino, Division of Gastroenterology, Department of Surgery, Oncology and Gastroenterology, University of Padua, Via Giustiniani 2, Padua 35121, Italy. Tel: +39-498217749, E-mail: edoardo.savarino@unipd.it
The introduction of biological drugs has radically changed the therapeutic approach and management of IBD patients. ${ }^{2-4}$ These drugs aim to achieve clinical, biochemical and endoscopic remission, therefore reducing the need for systemic steroids, hospitalization and surgery. In particular, anti-tumor necrosis factor (TNF) therapies such as infliximab, adalimumab (ADA), and golimumab have greatly improved treatment expectations in IBD patients refractory or intolerant to standard treatments, allowing achievement and maintenance of clinical remission and mucosal healing. ${ }^{5-11}$ Moreover, these drugs are able to halt IBD progression, improve the quality of life of the patients and control the disability associated with IBD., ${ }^{2,12-17}$ 
However, a fraction of patients experience primary nonresponse or loss of response to anti-TNF treatment, hence, a biodrug with a different mechanism of action, vedolizumab (VDZ), has been developed.$^{18} \mathrm{VDZ}$ is a monoclonal antibody that targets $\alpha 4 \beta 7$-integrin expressed in a subset of T- lymphocytes, preventing their endothelial adherence and migration towards the bowel mucosa. It is able to induce clinical remission and mucosal healing, even in the proportion of patients who experienced loss of response to anti-TNF drugs. ${ }^{19-21}$

Although the safety profile of these drugs is good, some studies have shown an increased risk of infections and in some cases of neoplasia, especially lymphomas and non-melanoma skin cancers. ${ }^{22-26}$ Apparently, these risks have been detected mostly in patients undergoing anti-TNF therapy, mainly when combination therapy with azathioprine was used and to a lesser extent in those taking VDZ. ${ }^{27-29}$ A previous nationwide population-based study including 190,694 patients with IBD found that anti-TNF monotherapy and combination therapy with thiopurines were associated with an increased risk of serious infection (hazard ratio [HR], 1.71; 95\% confidence interval [CI], 1.56-1.88). ${ }^{29}$ On the other hand, a recent report from the GEMINI long-term safety study reported that VDZ treatment was associated with relatively low rates of serious infections (18.0 and 33.6/1,000 person-years [PY] in UC and CD, respectively). ${ }^{30}$ In the most recent VARSITY study, a doubleblind, head to head investigation evaluating the effectiveness of VDZ as compared to ADA in UC, VDZ was associated with lower rates of overall and serious infections than ADA (23.4/ 100 PY vs. 34.6/100 PY and 1.6/100 PY vs. 2.2/100 PY, respectively). ${ }^{31}$

Currently, literature is lacking in studies comparing the incidence of adverse events (AEs) in patients treated with these 2 drugs in a real-life scenario. Therefore, our primary aim was to compare the AEs causing drug interruption in patients taking ADA to those taking VDZ. As secondary aim, we aimed to compare any other side effects (not causing drug interruption) in the first year of treatment between the 2 drugs.

\section{METHODS}

\section{Methods}

In this observational monocenter study, between January 2016 and September 2019, data on AEs from consecutive patients with IBD (i.e., with histologically confirmed diagnosis) who underwent treatment with ADA and VDZ at the IBD Unit of Padua University were collected. An open cohort approach was used, meaning that individuals could enter and exit the study at different time points. The study-start date was defined as the date on which biologic therapy was started, whereas the study-end date was considered as the earliest event between the last outpatient visit and the date of the AE that caused drug withdrawal. AEs were collected from medical records and divided into 6 categories: infusion reaction, injection-site reaction, infection, noninfectious extraintestinal events (pulmonary, cardiac, neurologic, dermatological, and articular AEs, etc), malignancy, and death. Information on biologic optimization (any type of optimization) during the study period was extracted from medical records. Initially, patients were treated according to international European Crohn's and Colitis Organisation guidelines ${ }^{3,32}$ and drugs were administered at standard dosage to all patients: VDZ, $300 \mathrm{mg}$ intravenously at zero, 2, and 6 weeks, then every 8 weeks; ADA, $160 \mathrm{mg}$, $80 \mathrm{mg}$, and then 40 mg every 2 weeks. However, during outpatient followup visits (post-induction, 6 months, 12 months or in case of disease recurrence) physicians (F.Z. and E.V.S) decided whether or not to optimize the drug based on clinical, biochemical and/or endoscopic response. The dosage available for therapeutic optimization was $300 \mathrm{mg}$ every 6 or 4 weeks for VDZ and $40 \mathrm{mg}$ every week or $80 \mathrm{mg}$ every 2 weeks for ADA.

Data on type and extension of IBD (CD or UC), sex, age at diagnosis, smoking status, and disease duration were collected, together with information on previous therapies. In particular, we collected data on previous biologic therapies (Supplementary Table 1) or whether immunosuppressive or steroid therapy were prescribed in the year preceding the start of the biologic therapy or if immunosuppressive and/or steroid were ongoing at the study start.

For our secondary aim, we collected any AEs, which did not cause drug interruption and occurred in the first year of the drug intake. For this analysis, the study-end date was considered as the earliest event between the last outpatient visit and the date of the first $\mathrm{AE}$, which had not caused drug withdrawal, occurred in the first year of treatment.

\section{Ethical Approval}

The study was approved by the Ethics Committee of Padua University as part of a larger study aimed to evaluate disease course and characteristics of IBD patients from the introduction of biologics in clinical practice (No. 3312/AO/14). Written informed consent was obtained from all eligible participants or their legal representatives before participation. 
Table 1. Demographic and Clinical Characteristics in Adalimumab and Vedolizumab Cohorts

\begin{tabular}{|c|c|c|c|}
\hline \multirow{2}{*}{ Characteristics } & \multicolumn{2}{|c|}{ Treatment } & \multirow{2}{*}{$P$-value } \\
\hline & ADA $(n=93)$ & VDZ $(n=145)$ & \\
\hline Disease & & & 0.040 \\
\hline Ulcerative colitis & $27(29.0)$ & $61(42.1)$ & \\
\hline Crohn's disease & $66(71.0)$ & $84(57.9)$ & \\
\hline Age at diagnosis (yr) & $31.80 \pm 13.08$ & $35.50 \pm 17.40$ & 0.080 \\
\hline Age study group & & & 0.110 \\
\hline$\leq 35 \mathrm{yr}$ & $24(25.8)$ & $30(20.7)$ & \\
\hline $36-54 \mathrm{yr}$ & $51(54.8)$ & $69(47.6)$ & \\
\hline$\geq 55 \mathrm{yr}$ & $18(19.4)$ & $46(31.7)$ & \\
\hline Sex & & & 0.850 \\
\hline Male & $56(60.2)$ & $89(61.4)$ & \\
\hline Female & $37(39.8)$ & $56(38.6)$ & \\
\hline Previous biologic therapy & & & $<0.001$ \\
\hline None & $44(47.3)$ & $20(13.8)$ & \\
\hline 1 Biologic therapy & $46(49.4)$ & $40(26.9)$ & \\
\hline$\geq 2$ Biologic therapies & $3(3.2)$ & $85(59.3)$ & \\
\hline \multicolumn{4}{|l|}{ Time from the last biologic therapy } \\
\hline$\leq 6 \mathrm{mo}$ & $39(41.9)$ & $77(53.1)$ & 0.090 \\
\hline Indication to therapy & & & 0.490 \\
\hline Active disease & $85(91.4)$ & $131(90.3)$ & \\
\hline Post-surgery & $1(1.1)$ & $5(3.4)$ & \\
\hline Intolerant to previous biologic therapy & $7(7.5)$ & $9(6.2)$ & \\
\hline Azathioprine in the year before the start date (no ongoing) & & & 0.300 \\
\hline No & $85(91.4)$ & $127(87.6)$ & \\
\hline Yes & $8(8.6)$ & $18(12.4)$ & \\
\hline Steroids in the year before the start date (no ongoing) & & & 0.600 \\
\hline No & $71(76.3)$ & $115(79.3)$ & \\
\hline Yes & $22(23.6)$ & $30(20.7)$ & \\
\hline Azathioprine ongoing & & & 0.600 \\
\hline No & $75(80.6)$ & $121(83.4)$ & \\
\hline Yes & $18(19.3)$ & $24(16.5)$ & \\
\hline Dosage $(\mathrm{mg})$ & $133.33 \pm 38.35$ & $131.25 \pm 38.48$ & 0.860 \\
\hline Steroid ongoing & & & 0.010 \\
\hline No & $72(77.4)$ & $90(62.1)$ & \\
\hline Yes & $21(22.6)$ & $55(37.9)$ & \\
\hline Dosage $(\mathrm{mg})$ & $18.33 \pm 16.07$ & $19.81 \pm 17.29$ & 0.730 \\
\hline Optimization therapy & & & 0.010 \\
\hline Yes & $28(30.1)$ & $67(46.2)$ & \\
\hline
\end{tabular}

Values are presented as number (\%) or mean \pm standard deviation.

ADA, adalimumab; VDZ, vedolizumab. 


\section{Statistical Analysis}

The different types of first AE were expressed as percentages for each class of biologic. Overall, AE rates per $100 \mathrm{PY}$ among subjects using $\mathrm{ADA}$ and VDZ were calculated. Cox regression models were used to estimate the HRs of the AEs between the different biologic therapies (VDZ vs. ADA). All HRs were adjusted for all covariates which were statistically significantly different between groups at baseline. STATA 11 was used for statistical analysis software (Stata Corp., College Station, TX, USA).

\section{RESULTS}

A total of 238 consecutive patients with IBD (88 UC and 150 CD) were included, $61 \%$ of whom were male. Among the study population, 93 patients commenced treatment with ADA and 145 with VDZ at the study start. Study population characteris- tics are summarized in Table 1. In particular, the ADA group included 66 patients with $\mathrm{CD}(71.0 \%)$ compared to 84 patients with CD (57.9\%) in VDZ group $(P=0.04), 44$ patients (47.3\%) treated with ADA were naïve to biologics compared to 20 (13.8\%) of those treated with VDZ, and only 3 of them (3.2\%) had already taken more than 2 biologics compared to 85 (59.3\%) in the VDZ group $(P<0.001)$. Finally, more VDZ patients were on steroid therapy and underwent drug optimization compared to patients treated with ADA (both $P=0.01$ ).

\section{AEs Causing Withdrawal of Therapy}

Median follow-up time was 1.24 years (range, $0.2-3$ years) for ADA and 1.3 years (range, $0.9-3$ years) for VDZ. Altogether, 328.6 PY on-drug were included: 121.3 PY for ADA patients and 207.3 PY for VDZ patients. A total of 16 ADA patients (17.2\%) and 11 VDZ patients (7.6\%) had AEs causing drug interruption during the study period $(P=0.02)$. Most of the AEs were

Table 2. Adverse Events Causing Withdrawal of Therapy in Patients Treated with ADA and VDZ

\begin{tabular}{|c|c|c|}
\hline AEs causing withdrawal & $\operatorname{ADA}(n=93)$ & $\operatorname{VDZ}(n=145)$ \\
\hline Total $^{a}$ & $16(17.2)$ & $11(7.6)$ \\
\hline \multirow[t]{2}{*}{ Infusion reaction } & $1(6.2)$ & $2(18.2)$ \\
\hline & · Angioedema & $\begin{array}{l}\text { - Hypertensive crisis during infusior } \\
\text { - Post-infusion lipothymia }\end{array}$ \\
\hline Injection-site reaction & $1(6.2)$ & 0 \\
\hline \multirow[t]{6}{*}{ Infection } & $5(31.2)$ & $3(27.3)$ \\
\hline & - Recurrent otitis & - Bronchitis \\
\hline & Pyelonephritis & · Cytomegalovirus reactivation \\
\hline & - Bilateral pneumonia & - Cough, dyspnea \\
\hline & Cough, dyspnea & \\
\hline & · Herpes simplex virus reactivation & \\
\hline \multirow[t]{7}{*}{ Noninfectious extraintestinal events } & $7(43.7)$ & $6(54.5)$ \\
\hline & · Headache and joint pain & · Joint pain worsening and psoriasis \\
\hline & - Chronic fatigue syndrome & · Psoriatic lesions \\
\hline & - Dermatitis and joint pain & - Urticaria and rash \\
\hline & - Psoriatic-like rash & $\cdot$ Heartbeat \\
\hline & - Thrombophlebitis & - Anxiety and panic attacks \\
\hline & · Alopecia (×2) & · Joint pain worsening \\
\hline \multirow[t]{3}{*}{ Malignancy } & $2(12.5)$ & 0 \\
\hline & · Melanoma in situ & \\
\hline & $\begin{array}{l}\text { - T-lymphoproliferative disease with a high } \\
\text { degree of malignancy }\end{array}$ & \\
\hline Death & 0 & 0 \\
\hline
\end{tabular}

Values are presented as the number (\%).

${ }^{a} A D A$ vs. VDZ, $P=0.02$.

AEs, adverse events; ADA, adalimumab; VDZ, vedolizumab. 


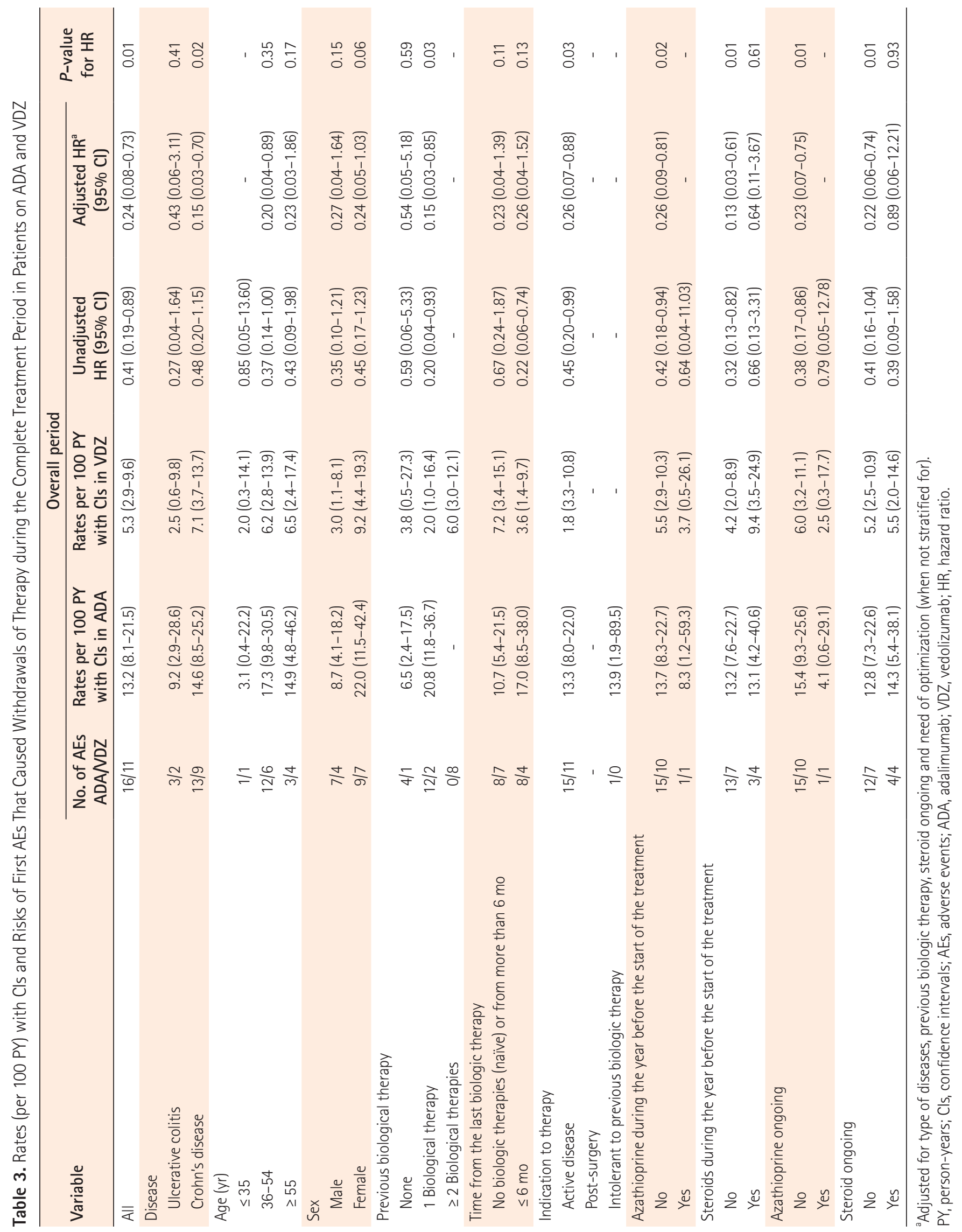


noninfectious extraintestinal events (43.7\% in ADA and 54.5\% in VDZ) while infections accounted for $31.2 \%$ of the AEs in patients treated with ADA and $27.3 \%$ in those treated with VDZ. One ADA patient and 2 VDZ patients experienced infusion reactions. Two patients treated with ADA had malignancy complications: 1 melanoma in situ and 1 lymphoma. Details on these AEs are reported in Table 2. The incidence rate of AEs causing withdrawal of therapy was 13.2 per $100 \mathrm{PY}$ for ADA and 5.3 per $100 \mathrm{PY}$ for VDZ, corresponding to a $76 \%$ lower risk in patients in VDZ compared to patients in ADA after adjustment for type of disease, being biologic naïve, ongoing steroid use and having undergone drug optimization (adjusted HR, $0.24 ; 95 \%$ CI, $0.08-0.73 ; P=0.01)$. This reduced risk was confirmed in patients aged more than 35 years, in patients with experience of more than 2 biologics, in those with active disease, and in both those who had not taken azathioprine and steroids in the year before and those who did not take

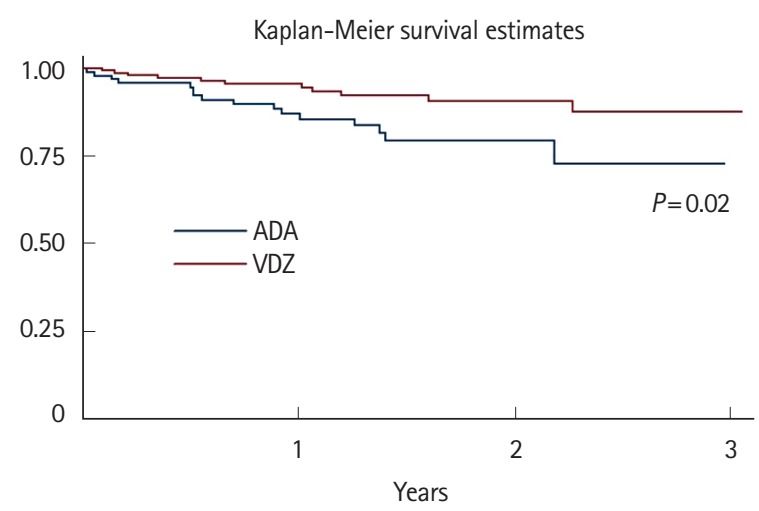

Fig. 1. Kaplan-Meier of adverse events which caused withdrawal of therapy in adalimumab (ADA) patients and in vedolizumab (VDZ) patients during the study period. them during the study (Table 3 ).

Fig. 1 shows the probability of AEs causing drug withdrawal in the 2 populations.

\section{AEs Which Did Not Cause Withdrawal of Therapy (during the First Year)}

Considering the first year of treatment, we observed 34 subjects treated with ADA (36.5\%) having at least $1 \mathrm{AE}$ and 57 (39.3\%) among those taking VDZ $(P=0.67)$ (Table 4). A second AE not causing drug interruption was observed in the first year in $5(5.4 \%)$ subjects with ADA and in 13 (9.0\%) subjects with VDZ $(P=0.44)$. Infections accounted for $29.4 \%(n=10)$ of the AEs in patients treated with ADA and $38.6 \%(\mathrm{n}=22)$ in those treated with VDZ $(P=0.90)$. The most common infections in ADA patients were respiratory tract infections following by dental abscesses, a case of pityriasis, 1 each of oral candidiasis and gastroenteritis. In the VDZ group, we observed

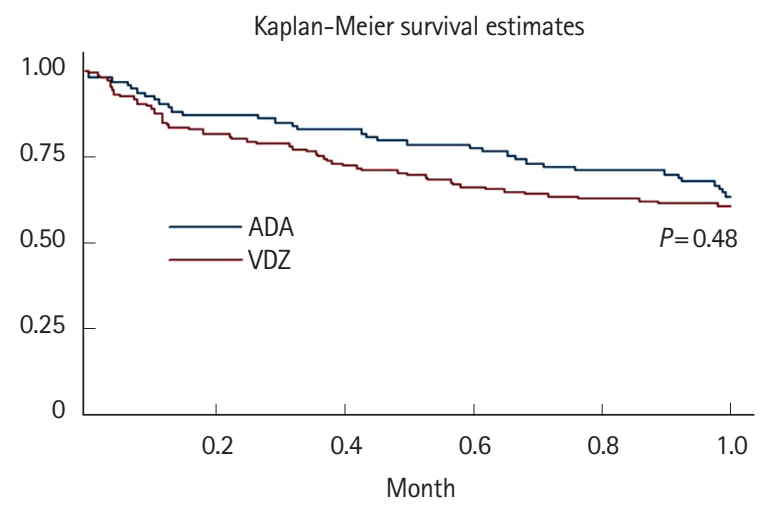

Fig. 2. Kaplan-Meier of adverse events which did not cause withdrawal of therapy in adalimumab (ADA) patients and in vedolizumab (VDZ) patients in the first year of treatment.

Table 4. Adverse Events which Did Not Cause Drug Interruption during the First Year of Treatment

\begin{tabular}{lccc}
\hline Variable & ADA $(\mathrm{n}=93)$ & VDZ $(\mathrm{n}=145)$ & $P$-value \\
\hline First AE not causing withdrawal & $34(36.5)$ & $57(39.3)$ & 0.67 \\
Infusion reaction & $3(8.8)$ & $7(12.3)$ & \\
Injection-site reaction & $1(2.9)$ & 0 & \\
Infection & $10(29.4)$ & $22(38.6)$ & \\
Noninfectious extraintestinal events & $20(58.8)$ & $28(49.1)$ & \\
Malignancy & 0 & 0 & \\
Death & 0 & 0 & 0.44 \\
Second AE not causing withdrawal & $5(5.4)$ & $13(9.0)$ & 0.80 \\
Third AE not causing withdrawal & $1(1.1)$ & $2(1.4)$ &
\end{tabular}

Values are presented as the number (\%).

AEs, adverse events; ADA, adalimumab; VDZ, vedolizumab. 
respiratory tract infections, infectious gastroenteritis, Herpes simplex virus and cytomegalovirus reactivations. Regarding noninfectious AEs the most frequent were rash, joint pain and asthenia.

The incidence rate of AEs which did not cause withdrawal of therapy was not statistically different between ADA and VDZ (45.7/100 PY vs. 54.2/100 PY, respectively) (Supplementary Table 1), except in males where we observed a lower risk of AEs in those treated with VDZ than those treated with ADA (adjusted HR, 0.44; 95\% CI, 0.19-0.97).

Fig. 2 shows the probability of AEs in the 2 populations during the first year of treatment.

\section{DISCUSSION}

While the first head-to-head trials comparing the effectiveness of various biological therapies for IBD patients have started to be published, ${ }^{10,11,33}$ studies focusing on the incidence of AEs of these compounds are still scarce. Overall, the safety profile of these drugs is high, but evidence of AEs, such as infections and, in some cases, the onset of malignancies has been reported. ${ }^{34,35}$ Thus, we conducted a study in a real-life scenario, which aimed to compare the safety profile of the VDZ to ADA. We observed that $17.2 \%$ of patients taking ADA and $7.6 \%$ of patients taking VDZ had AEs causing drug interruption during the study period. In particular, among these, infections accounted for 31.2\% of the AEs in patients treated with ADA and $27.3 \%$ in those treated with VDZ. Two patients treated with ADA had malignancy diagnosed: melanoma in situ and lymphoma. Overall, the incidence rate of AEs causing withdrawal of therapy was 13.2 per 100 PY for ADA and 5.3 per 100 PY for VDZ, corresponding to a $76.0 \%$ lower risk in patients taking VDZ compared to patients taking ADA. Moreover, we found that $36.5 \%$ of patients taking ADA and 39.3\% patients taking VDZ had at least $1 \mathrm{AE}$ which did not cause drug interruption, without a statistically significant difference between the 2 drugs. Among the latter, we observed a higher percentage of infections in VDZ group compared to ADA group (38.6\% vs. $29.4 \%$ ), which may be due to the higher percentage of steroids ongoing and of optimization rates in VDZ group; however, this difference is not statistically significantly different $(P=0.90)$.

To date, the VARSITY study has been the only randomized controlled trial (RCT) to compare the efficacy and safety of VDZ and ADA. ${ }^{31}$ This recently published study by Sands et $\mathrm{al}^{3 .}{ }^{31}$ showed that $62.7 \%$ of patients with ADA and $69.2 \%$ of patients with VDZ experienced at least $1 \mathrm{AE}$, while AEs that caused the suspension of therapy occurred in $6.5 \%$ and in $4.4 \%$ of patients, respectively. They reported that the incidence rate of infections was also higher in ADA (34.6/100 PY) compared to VDZ (23.4/100 PY). It is worth underlining the fact that the VARSITY study population was well balanced between the 2 drugs with similar percentages of naïve and not naïve patients, whereas our cohorts were less homogeneous. In particular, more patients in the ADA group were naïve compared to those in the VDZ group ( $47.3 \%$ vs. $13.8 \%, P<0.001$, respectively) which was to be expected given that VDZ was approved for use after ADA and primarily in patients who failed anti-TNF- $\alpha$ therapy. Also, more VDZ patients were on steroid therapy and needed drug optimization compared to patients treated with ADA (both $P=0.01$ ), reflecting what is generally found in daily clinical practice. However, the adjusted analysis should reduce the effect of these differences.

To the best of our knowledge, few studies have reported estimates of the incidence rates of AEs causing withdrawal of biological therapies in moderate to severe IBD patients. For instance, a Japanese study ${ }^{36}$ estimated an incidence rate of 12.4 per $100 \mathrm{PY}$ of AEs leading of discontinuation of use of ADA in moderate to severe UC patients, which is quite similar to that reported in our study (13.2/100 PY). On the other hand, in patients with $\mathrm{CD}$ treated with $\mathrm{ADA}$, our study reported an incidence rate of AEs leading to drug discontinuation of 14.6 per $100 \mathrm{PY}$, which is lower than the figure of 22.1 per $100 \mathrm{PY}$ in patients in ADA monotherapy published by Colombel et al. ${ }^{37}$ In that study, the incidence rate of AE became higher (29.2/100 PY) when considering patients on combination therapy with immunomodulators, ${ }^{37}$ a finding we were unable to replicate. Conversely, considering the general incidence rate of the first $\mathrm{AE}$ in ADA patients reported in the literature, it ranges from 40.4 per $100 \mathrm{PY}^{38}$ to 76.1 per $100 \mathrm{PY}^{39}$ while in our study it was 45.7 per $100 \mathrm{PY}$, and therefore in line with that reported by other authors.

Among our patients treated with VDZ, 39.3\% developed at least $1 \mathrm{AE}$ and $7.6 \%$ experienced an $\mathrm{AE}$ which caused treatment discontinuation. Regarding the percentage developing at least $1 \mathrm{AE}$, our results showed lower percentages than those reported in a meta-analysis by Moćko et al. ${ }^{40}$ which included only CD patients from the 2 RCTs GEMINI II and GEMINI III (39.3\% in our study vs. $56.0 \%$ from this pooled analysis). While, we reported a slightly higher percentage of AEs causing withdrawal of therapy compared to those of these authors $(7.6 \%$ vs. $2 \%$, respectively). ${ }^{40}$ The incidence rate of the first $\mathrm{AE}$ in our VDZ population, considering the entire time at risk, was 54.2 
per $100 \mathrm{PY}$. This figure, however, differs greatly from what emerged from a study by Colombel et al.," ${ }^{41}$ reporting an integrated summary of data from 6 double-blind or open-label trials of IBD patients treated with VDZ, which reports an incidence rate of 247.8 per $100 \mathrm{PY}$. This is perhaps unsurprising since our data are from routine clinical practice where the recognition and reporting of minor AEs are likely to be slower than in the trials data reported by Colombel. However, the authors ${ }^{41}$ reported an incidence rate of AEs causing treatment discontinuation of 6 per $100 \mathrm{PY}$, which is similar to what we found (5.3/ $100 \mathrm{PY})$.

Our study focusing on the comparison of the safety profile between ADA and VDZ represents a novelty. Only few studies comparing the safety of VDZ with other anti-TNF biologics have been published and among these, only 1 is a direct comparison. ${ }^{31}$ The meta-analyses found are based mainly on RCTs ${ }^{41,42}$ conducted in selected populations rather than on non-selective cohorts in real life. Therefore, these studies may not reflect the very heterogeneous population found in clinical practice. Moreover, in many studies, the categorization of AEs occurred on the basis of their seriousness, without a distinction of those that led to the discontinuation of treatment. The direct comparison conducted by Sands et al., ${ }^{31}$ regarding the AEs that caused the interruption of the trial, only provided the percentages of patients in whom they occurred, but did not provide the incidence rate. Moreover, we were able to correct the risk data for those variables that could indirectly influence it.

However, this study has also some limitations. Given the retrospective design, the collection of AEs took place on the basis of the medical records relating to the follow-up outpatient visits, with the consequent risk that they might have failed to report AEs that occurred in the period between visits or that these have not been adequately reported. In addition, this is a single center study and data were collected by a single operating unit. Therefore, the study population was representative of a single geographical area. Moreover, the time of follow-up was relatively limited, not allowing the evaluation of events that could arise long after the start of biological therapy. For our secondary aim, we decided to limit our analysis to the first year of treatment because during the first year of therapy patients are followed more strictly at our center with at least 3 outpatient clinics and therefore lower risk of missing data. Finally, since the study was conducted in real life, the study population was not as homogeneous as in registration studies, and some differences in the distribution of the population between the group of patients receiving VDZ and that receiving $\mathrm{ADA}$ were detect- ed. Though these will inevitably have biased the findings with respect to the risk of AEs, nonetheless our findings give a far clearer picture of the risks likely to be encountered in clinical practice where VDZ and ADA are used in the manner in which they are used in our practice.

The comparison with biological drugs with different mechanisms of action, including anti-TNF drugs and anti-integrin treatments, represents a step forward compared to what is already known, which supports the choice of the drug in particular categories at risk. Surely, real-life head-to-head studies, comparing different molecules, are still necessary to further explore the safety profile of these drugs.

\section{ADDITIONAL INFORMATION}

\section{Funding Source}

The authors received no financial support for the research, authorship, and/or publication of this article.

\section{Conflict of Interest}

Savarino EV has received lecture or consultancy fees from Takeda, Merck \& Co, Bristol-Myers Squibb, AbbVie, Amgen, Novartis, Fresenius Kabi, Sandoz, Sofar, Janssen. Card T was previously married to a subsequent employee of Takeda. Zingone F has received lecture fees from Takeda, Janssen, Norgine. The other authors declare that they have no conflicting interests.

\section{Author Contribution}

Conceptualization: Zingone F. Drafting study: Barberio B, Zingone F. Data collection: Barberio B, Baldisser F, Gubbiotti A, Massimi D, Ghisa M. Data analysis and interpretation: Zingone F, Canova C, Card T. Writing - original draft: Barberio B, Savarino EV, Zingone F. Writing - review and editing: all authors. Approval of final manuscript: all authors.

\section{Others}

The data underlying this study are available within the manuscript and supplementary materials.

\section{ORCID}

Barberio B

https://orcid.org/0000-0002-3164-8243

Savarino EV https://orcid.org/0000-0002-3187-2894

Card T https://orcid.org/0000-0003-2555-2250

Canova C https://orcid.org/0000-0001-7027-7935

Baldisser F https://orcid.org/0000-0001-6734-1131 
Gubbiotti A

https://orcid.org/0000-0002-0174-8729

Massimi D

Ghisa M

Zingone $\mathrm{F}$ https://orcid.org/0000-0002-0926-514X https://orcid.org/0000-0002-6026-5639 https://orcid.org/0000-0003-1133-1502

\section{Supplementary Material}

Supplementary materials are available at the Intestinal Research website (https://www.irjournal.org).

\section{REFERENCES}

1. Maaser C, Sturm A, Vavricka SR, et al. ECCO-ESGAR Guideline for Diagnostic Assessment in IBD Part 1: Initial diagnosis, monitoring of known IBD, detection of complications. J Crohns Colitis 2019;13:144-164.

2. Pineton de Chambrun G, Blanc P, Peyrin-Biroulet L. Current evidence supporting mucosal healing and deep remission as important treatment goals for inflammatory bowel disease. Expert Rev Gastroenterol Hepatol 2016;10:915-927.

3. Torres J, Bonovas S, Doherty G, et al. ECCO guidelines on therapeutics in Crohn's disease: medical treatment. J Crohns Colitis 2020;14:4-22.

4. Barberio B, Black CJ, Savarino EV, Ford AC. Ciclosporin or infliximab as rescue therapy in acute glucorticosteroid-refractory ulcerative colitis: systematic review and network meta-analysis. J Crohns Colitis 2021;15:733-741.

5. Dignass A, Lindsay JO, Sturm A, et al. Second European evidence-based consensus on the diagnosis and management of ulcerative colitis part 2: current management. J Crohns Colitis 2012;6:991-1030.

6. Nielsen $\mathrm{OH}$, Ainsworth MA. Tumor necrosis factor inhibitors for inflammatory bowel disease. N Engl J Med 2013;369:754762.

7. Rutgeerts P, Sandborn WJ, Feagan BG, et al. Infliximab for induction and maintenance therapy for ulcerative colitis. N Engl J Med 2005;353:2462-2476.

8. Sandborn WJ, van Assche G, Reinisch W, et al. Adalimumab induces and maintains clinical remission in patients with moderate-to-severe ulcerative colitis. Gastroenterology 2012;142: 257-265

9. Sandborn WJ, Feagan BG, Marano C, et al. Subcutaneous golimumab maintains clinical response in patients with moderate-to-severe ulcerative colitis. Gastroenterology 2014;146:96109.

10. Barberio B, Zingone F, D'Incà R, et al. Infliximab originator, infliximab biosimilar, and adalimumab are more effective in
Crohn's disease than ulcerative colitis: a real-life cohort study. Clin Transl Gastroenterol 2020;11:e00177.

11. Barberio B, Zingone F, Frazzoni L, et al. Real-life comparison of different anti-TNF biologic therapies for ulcerative colitis treatment: a retrospective cohort study. Dig Dis 2021;39:1624.

12. Marinelli C, Savarino E, Inferrera M, et al. Factors influencing disability and quality of life during treatment: a cross-sectional study on IBD patients. Gastroenterol Res Pract 2019;2019: 5354320.

13. Marinelli C, Zingone F, Inferrera M, et al. Factors associated with disability in patients with ulcerative colitis: a cross-sectional study. J Dig Dis 2020;21:81-87.

14. Marinelli C, Savarino EV, Marsilio I, et al. Sleep disturbance in inflammatory bowel disease: prevalence and risk factors-a cross-sectional study. Sci Rep 2020;10:507.

15. Barberio B, Zingone F, Savarino EV. Inflammatory bowel disease and sleep disturbance: as usual, quality matters. Dig Dis Sci 2021;66:3-4

16. Bodini G, Giannini EG, De Maria C, et al. Anti-TNF therapy is able to stabilize bowel damage progression in patients with Crohn's disease: a study performed using the Lémann Index. Dig Liver Dis 2017;49:175-180.

17. Barberio B, Zamani M, Black CJ, Savarino EV, Ford AC. Prevalence of symptoms of anxiety and depression in patients with inflammatory bowel disease: a systematic review and metaanalysis. Lancet Gastroenterol Hepatol 2021;6:359-370.

18. Zingone F, Barberio B, Compostella F, et al. Good efficacy and safety of vedolizumab in Crohn's disease and ulcerative colitis in a real-world scenario. Therap Adv Gastroenterol 2020;13: 1756284820936536.

19. Feagan BG, Rutgeerts P, Sands BE, et al. Vedolizumab as induction and maintenance therapy for ulcerative colitis. N Engl J Med 2013;369:699-710.

20. Sandborn WJ, Feagan BG, Rutgeerts P, et al. Vedolizumab as induction and maintenance therapy for Crohn's disease. N Engl J Med 2013;369:711-721.

21. Scribano ML. Vedolizumab for inflammatory bowel disease: from randomized controlled trials to real-life evidence. World J Gastroenterol 2018;24:2457-2467.

22. Colombel JF, Sandborn WJ, Rutgeerts P, et al. Adalimumab for maintenance of clinical response and remission in patients with Crohn's disease: the CHARM trial. Gastroenterology 2007; 132:52-65.

23. Murdaca G, Spanò F, Puppo F. Selective TNF- $\alpha$ inhibitor-induced injection site reactions. Expert Opin Drug Saf 2013;12: 
$187-193$

24. Cantini F, Niccoli L, Goletti D. Adalimumab, etanercept, infliximab, and the risk of tuberculosis: data from clinical trials, national registries, and postmarketing surveillance. J Rheumatol Suppl 2014;91:47-55.

25. Osterman MT, Sandborn WJ, Colombel JF, et al. Increased risk of malignancy with adalimumab combination therapy, compared with monotherapy, for Crohn's disease. Gastroenterology 2014;146:941-949.

26. Barberio B, Gubbiotti A, Albertoni L, Ghisa M, Savarino E. Gastrointestinal: an unusual rectal finding in a patient with ulcerative colitis. J Gastroenterol Hepatol 2020;35:179.

27. Shah ED, Farida JP, Siegel CA, Chong K, Melmed GY. Risk for overall infection with anti-TNF and anti-integrin agents used in IBD: a systematic review and meta-analysis. Inflamm Bowel Dis 2017;23:570-577.

28. Card T, Ungaro R, Bhayat F, Blake A, Hantsbarger G, Travis S. Vedolizumab use is not associated with increased malignancy incidence: GEMINI LTS study results and post-marketing data. Aliment Pharmacol Ther 2020;51:149-157.

29. Kirchgesner J, Lemaitre M, Carrat F, Zureik M, Carbonnel F, Dray-Spira R. Risk of serious and opportunistic infections associated with treatment of inflammatory bowel diseases. Gastroenterology 2018;155:337-346.

30. Loftus EV Jr, Feagan BG, Panaccione R, et al. Long-term safety of vedolizumab for inflammatory bowel disease. Aliment Pharmacol Ther 2020;52:1353-1365.

31. Sands BE, Peyrin-Biroulet L, Loftus EV Jr, et al. Vedolizumab versus adalimumab for moderate-to-severe ulcerative colitis. N Engl J Med 2019;381:1215-1226.

32. Harbord M, Eliakim R, Bettenworth D, et al. Third European evidence-based consensus on diagnosis and management of ulcerative colitis. part 2: current management. J Crohns Colitis $2017 ; 11: 769-784$.

33. Renna S, Mocciaro F, Ventimiglia M, et al. A real life comparison of the effectiveness of adalimumab and golimumab in moderate-to-severe ulcerative colitis, supported by propensi- ty score analysis. Dig Liver Dis 2018;50:1292-1298.

34. Luthra P, Peyrin-Biroulet L, Ford AC. Systematic review and meta-analysis: opportunistic infections and malignancies during treatment with anti-integrin antibodies in inflammatory bowel disease. Aliment Pharmacol Ther 2015;41:1227-1236.

35. Chupin A, Perduca V, Meyer A, Bellanger C, Carbonnel F, Dong C. Systematic review with meta-analysis: comparative risk of lymphoma with anti-tumour necrosis factor agents and/or thiopurines in patients with inflammatory bowel disease. Aliment Pharmacol Ther 2020;52:1289-1297.

36. Suzuki Y, Motoya S, Hanai H, et al. Four-year maintenance treatment with adalimumab in Japanese patients with moderately to severely active ulcerative colitis. J Gastroenterol 2017;52:1031-1040.

37. Colombel JF, Jharap B, Sandborn WJ, et al. Effects of concomitant immunomodulators on the pharmacokinetics, efficacy and safety of adalimumab in patients with Crohn's disease or ulcerative colitis who had failed conventional therapy. Aliment Pharmacol Ther 2017;45:50-62.

38. Loftus EV, Reinisch W, Panaccione R, et al. Adalimumab effectiveness up to six years in adalimumab-naïve patients with Crohn's disease: results of the PYRAMID registry. Inflamm Bowel Dis 2019;25:1522-1531.

39. Ogata H, Watanabe M, Matsui T, et al. Safety of adalimumab and predictors of adverse events in 1693 Japanese patients with Crohn's disease. J Crohns Colitis 2016;10:1033-1041.

40. Moćko P, Kawalec P, Smela-Lipińska B, Pilc A. Effectiveness and safety of vedolizumab for treatment of Crohn's disease: a systematic review and meta-analysis. Arch Med Sci 2016;12: 1088-1096.

41. Colombel JF, Sands BE, Rutgeerts P, et al. The safety of vedolizumab for ulcerative colitis and Crohn's disease. Gut 2017;66: 839-851.

42. Moćko P, Kawalec P, Pilc A. Safety profile of biologic drugs in the therapy of Crohn disease: a systematic review and network meta-analysis. Pharmacol Rep 2016;68:1237-1243. 


\section{See "Incidence comparison of adverse events in patients with inflammatory bowel disease receiving different bio-} logic agents: retrospective long-term evaluation" on page 114-123.

Supplementary Table 1. Rates (per 100 PY) and Risks of Any First Adverse Events during the First Year of Treatment in Patients in ADA and VDZ

\begin{tabular}{|c|c|c|c|}
\hline Variable & $\begin{array}{l}\text { No. of events } \\
\text { ADA/VDZ }\end{array}$ & $\begin{array}{c}\text { Rates per } 100 \text { PY with } \\
\text { Cls in ADA }\end{array}$ & $\begin{array}{c}\text { Rates per } 100 \text { PY with } \\
\text { Cls in VDZ }\end{array}$ \\
\hline All & $34 / 57$ & $45.7(32.7-64.0)$ & $54.2(41.8-70.3)$ \\
\hline Ulcerative colitis & $11 / 25$ & $58.7(32.5-105.9)$ & $57.9(39.1-85.7)$ \\
\hline Crohn's disease & $23 / 32$ & $41.4(27.5-62.3)$ & $51.6(36.5-72.9)$ \\
\hline$\leq 35$ & $10 / 10$ & $52.8(28.4-92.2)$ & $43.7(23.5-81.3)$ \\
\hline $36-54$ & $18 / 27$ & $43.0(27.1-68.3)$ & $54.8(37.6-79.9)$ \\
\hline$\geq 55$ & $6 / 20$ & $44.1(19.8-98.2)$ & $60.5(39.0-93.7)$ \\
\hline \multicolumn{4}{|l|}{ Sex } \\
\hline \multicolumn{4}{|l|}{ Previous biological therapy } \\
\hline None & $15 / 4$ & $43.7(26.3-72.4)$ & $23.3(8.7-62.0)$ \\
\hline 1 Biological therapy & $18 / 10$ & $48.5(30.6-67.0)$ & $32.3(17.4-60.1)$ \\
\hline$\geq 2$ Biological therapies & $1 / 43$ & $34.5(4.8-245.2)$ & $75.3(55.9-101.6)$ \\
\hline \multicolumn{4}{|l|}{ Time from the last biological therapy } \\
\hline No biologic therapies (naïve) or from more than 6 mo & $18 / 25$ & $42.0(26.5-66.8)$ & $50.9(34.4-75.3)$ \\
\hline$\leq 6 \mathrm{mo}$ & $16 / 32$ & $50.7(31.0-82.7)$ & $57.1(40.4-80.1)$ \\
\hline \multicolumn{4}{|l|}{ Indication to therapy } \\
\hline Active disease & $30 / 52$ & $44.6(31.2-63.8)$ & $54.5(41.5-71.5)$ \\
\hline Post-surgery & - & - & - \\
\hline No & $26 / 43$ & $46.2(31.4-67.9)$ & $49.3(36.6-66.5)$ \\
\hline Yes & $8 / 14$ & $44.3(22.1-88.5)$ & 77.6 (45.9-130.9) \\
\hline \multicolumn{4}{|l|}{ Azathioprine ongoing } \\
\hline No & $28 / 46$ & $46.5(32.1-67.3)$ & $52.1(39.0-69.6)$ \\
\hline Yes & $6 / 11$ & $42.5(19.1-94.5)$ & $65.1(36.0-117.6)$ \\
\hline \multicolumn{4}{|l|}{ Steroids ongoing } \\
\hline No & $27 / 33$ & $46.2(31.7-67.4)$ & $50.4(35.8-60.9)$ \\
\hline Yes & $7 / 24$ & 43.9 (20.9-92.1) & $60.5(40.5-90.2)$ \\
\hline
\end{tabular}

PY, person-years; ADA, adalimumab; VDZ, vedolizumab; Cls, confidence intervals. 